Article

\title{
Interfacial Engineering of Attractive Pickering Emulsion Gel-Templated Porous Materials for Enhanced Solar Vapor Generation
}

\author{
Xiaoxiao Yan ${ }^{1,+}$, Baiheng $W u^{1,+}$, Qinglin Wu ${ }^{1,2}$, Li Chen 1,3,4, Fangfu Ye ${ }^{3,4,5}$ and Dong Chen ${ }^{1,2, *}$ \\ 1 College of Energy Engineering and State Key Laboratory of Fluid Power and Mechatronic Systems, \\ Zhejiang University, Hangzhou 310027, China; 21927067@zju.edu.cn (X.Y.); bhwu@zju.edu.cn (B.W.); \\ 22027098@zju.edu.cn (Q.W.); chenli20@ucas.ac.cn (L.C.) \\ 2 Department of Medical Oncology, The First Affiliated Hospital, School of Medicine, Zhejiang University, \\ Hangzhou 310003, China \\ 3 Wenzhou Institute, University of Chinese Academy of Sciences, Wenzhou 325001, China; fye@iphy.ac.cn \\ 4 Beijing National Laboratory for Condensed Matter Physics, Institute of Physics, Chinese Academy of \\ Sciences, Beijing 100190, China \\ 5 Oujiang Laboratory (Zhejiang Lab for Regenerative Medicine, Vision and Brain Health), \\ Wenzhou 325001, China \\ * Correspondence: chen_dong@zju.edu.cn \\ + These authors contribute equally to this work.
}

Citation: Yan, X.; Wu, B.; Wu, Q.; Chen, L.; Ye, F.; Chen, D. Interfacial Engineering of Attractive Pickering Emulsion Gel-Templated Porous Materials for Enhanced Solar Vapor Generation. Energies 2021, 14, 6077. https://doi.org/10.3390/en14196077

Academic Editor: Vijay Kumar Thakur

Received: 15 August 2021

Accepted: 9 September 2021

Published: 24 September 2021

Publisher's Note: MDPI stays neutral with regard to jurisdictional claims in published maps and institutional affiliations.

Copyright: (c) 2021 by the authors. Licensee MDPI, Basel, Switzerland. This article is an open access article distributed under the terms and conditions of the Creative Commons Attribution (CC BY) license (https:// creativecommons.org/licenses/by/ $4.0 /)$.

\begin{abstract}
Solar vapor generation is emerging as one of the most important sustainable techniques for harvesting clean water using abundant and green solar energy. The rational design of solar evaporators to realize high solar evaporation performances has become a great challenge. Here, a porous solar evaporator with integrative optimization of photothermal convention, water transport and thermal management is developed using attractive Pickering emulsions gels (APEG) as templated and followed by interfacial engineering on a molecular scale. The APEG-templated porous evaporators (APEG-TPEs) are intrinsically thermal insulation materials with a thermal conductivity = $0.039 \mathrm{~W} \cdot \mathrm{m}^{-1} \cdot \mathrm{K}^{-1}$. After hydrolysis, $t$-butyl groups on the inner-surface are transformed to carboxylic acid groups, making the inner-surface hydrophilic and facilitating water transport through the interconnected pores. The introduction of polypyrrole layer endows the porous materials with a high light absorption of $\sim 97 \%$, which could effectively convert solar irradiation to heat. Due to the versatility of the APEG systems, the composition, compressive modulus, porosity of APEG-TPEs could be well controlled and a high solar evaporation efficiency of $69 \%$ with an evaporation rate of $1.1 \mathrm{~kg} \cdot \mathrm{m}^{-2} \cdot \mathrm{h}^{-1}$ is achieved under simulated solar irradiation. The interface-engineered APEG-TPEs are promising in clean water harvesting and could inspire the future development of solar evaporators.
\end{abstract}

Keywords: solar evaporator; porous material; emulsion; gel; interfacial engineering

\section{Introduction}

Clean water shortage is a global issue, impeding sustainable development and threatening human survival [1-4]. Water purification via water evaporation using abundant solar energy is regarded as a green and sustainable technology to address the challenge of water shortage [5-10]. Recently, great efforts have been devoted to the developments of advanced solar evaporators with high utilization efficiency of solar irradiation for clean water harvesting. Sunlight absorption, heat transfer and water transport are the three main factors, which need to be optimized to improve the utilization efficiency of solar energy for water evaporation [11-14]. Generally, materials with a high absorption over the whole solar spectrum, such as plasmonic nanoparticles [15-18], carbon materials [19-22] or black polymers [23-26], are adopted as the surface layer. The heat absorbed from sunlight irradiation is then expected to heat up water to facilitate water evaporation, instead of transferring 
to the water reservoir. Therefore, thermal insulation materials are preferred to confine the heat at the surface and block undesired heat conduction to the water reservoir $[27,28]$. Meanwhile, water evaporation is usually localized at the surface and water should easily be transported to the surface via water pathway to facilitate water evaporation $[29,30]$.

To improve the solar evaporation efficiency, a lot of efforts have been dedicated to the design of solar evaporators. For example, a solar evaporator with a confined 2D water path on a polystyrene foam is developed [31]. Enabled by the confined 2D water path, the heat loss is minimized. A bilayer evaporator is designed by electrostatic spinning, where small holes are punched on the bottom insulation layer to direct water transport [32]. Inspired by the evapotranspiration of plants, carbonized mushrooms are made into solar evaporators, which possess unique natural structures of fibrous stipes [33]. As thermal management is playing an increasingly important role in improving the efficiency of solar energy utilization, commercialized thermal insulation materials, such as polystyrene foams, are also used in the rational design of solar evaporators. However, polystyrene foams are highly hydrophobic, which is adverse to water transport [34]. In addition, it is hard to engineer the microstructure of polystyrene foams and thus hard to construct interconnected water pathway for water transport, restricting the applications of polystyrene foams. Therefore, innovations of new materials and designs are highly desired.

To solve the paradox between thermal management and water transport, an integral solar evaporator based on the interfacial engineering of thermal insulation porous materials is developed. The porous materials are prepared using attractive Pickering emulsion gels (APEGs) as templates, which are a versatile system with tunable composition and porosity [35-37]. The APEG-templated porous evaporators (APEG-TPEs) are then hydrolyzed to facilitate water transport and coated with polypyrrole (PPy) to improve photothermal conversions. The optimized performances of APEG-TPEs could achieve a solar evaporation efficiency of $69 \%$ and an evaporation rate of $1.1 \mathrm{~kg} \cdot \mathrm{m}^{-2} \cdot \mathrm{h}^{-1}$ under simulated solar irradiation. The results show great potentials of the APEG-TPEs for the future developments of solar evaporators.

\section{Materials and Methods}

\subsection{Materials}

Shellac (wax free), styrene (St), divinyl benzene (DVB) and phenylbis(2,4,6-trimethylbenzoyl)phosphine oxide (photoinitiator 819) were purchased from Sigma-Aldrich. Trifluoroacetic acid (TFA) and pyrrole were obtained from Macklin Inc. $\alpha, \omega$-diamino-functionalized polystyrene $\left(\mathrm{NH}_{2}-\mathrm{PS}-\mathrm{NH}_{2}\right)$ with a molecular weight of $M_{\mathrm{W}} \sim 9000$ was synthesized via atom transfer radical polymerization using a dibromo-functionalized initiator and then nucleophilically substituted to yield diamino end groups. Ammonium persulfate (APS), $t$-butyl acrylate ( $t$-BA), dichloromethane $\left(\mathrm{CH}_{2} \mathrm{Cl}_{2}\right)$ and ethanol were purchased from Sinopharm Chemical Reagent Co., Ltd. The inhibitors in styrene and $n$-butyl acrylate monomers were removed by passing them through a basic alumina column before use. Deionized (DI) water was used throughout the study.

\subsection{Methods}

2.2.1. Preparation of Attractive Pickering Emulsion Gels-Templated Porous Evaporators (APEG-TPEs)

A series of photopolymerizable APEGs were prepared according to the reference [38]. Shellac nanoparticles (NPs) were prepared by flash nanoprecipitation. A total of $25 \mathrm{mg}$ shellac was dissolved in $1 \mathrm{~mL}$ ethanol. Then, $100 \mu \mathrm{L}$ of the shellac solution was quickly injected into $3 \mathrm{~mL}$ DI water using a 1-200 $\mu \mathrm{L}$ gel-loading pipet tip. As the ethanol quickly mixed with water, the shellac precipitated, forming NPs. The size of shellac NPs was $\mathrm{d} \sim 80 \mathrm{~nm}$. Shellac NPs were naturally carboxyl functionalized, which contained a lot of carboxyl groups on the surface.

In a typical APEG system, the concentration of shellac NPs in water was $0.83 \mathrm{mg} \cdot \mathrm{mL}^{-1}$ and the concentration of $\mathrm{NH}_{2}-\mathrm{PS}-\mathrm{NH}_{2}$ and photoinitiator 819 in the photopolymerizable oil 
were both $10 \mathrm{mg} \cdot \mathrm{mL}^{-1}$. The oil phase was consisted of $\mathrm{St}, t$-BA and DVB with a molar ratio of St:t-BA:DVB = 4:5:1. The volume ratio of oil to water was 1:4, 3:17 and 1:9 to prepare APEGs with porosity $=80 \%, 85 \%$ and $90 \%$, respectively. The mixtures were sheared into emulsions by shaking, which formed APEGs after a couple of minutes in stationary. The APEGs were then sealed in a home-made mold, which consisted of two glass plates spaced by a PDMS film with a square opening of $6 \times 8 \mathrm{~cm}$ and a thickness of $2 \mathrm{~mm}$. The mold was then placed under a UV lamp $(395 \mathrm{~nm}, 18 \mathrm{~W})$ to initiate the polymerization. After $4 \mathrm{~h}$, APEG-TPEs were washed with ethanol for 3 times and dried in a vacuum oven overnight.

\subsubsection{Hydrophilization of APEG-TPEs}

The hydrolysis of $t$-BA in a solution of TFA and $\mathrm{CH}_{2} \mathrm{Cl}_{2}$ was used to produce carboxylic acid groups on the inner-surface of the porous materials. The hydrolysis procedure could effectively hydrolyze $t$-BA within several hours, producing $t$-butyl trifluoroacetic acids. APEG-TPEs were sliced into $4 \mathrm{~cm}$-diameter circular pieces and was hydrolyzed at room temperature through immersion in a mixture of TFA $(2 \mathrm{~mL})$ and $\mathrm{CH}_{2} \mathrm{Cl}_{2}(20 \mathrm{~mL})$ for $48 \mathrm{~h}$. After hydrolysis, the samples were washed with $\mathrm{CH}_{2} \mathrm{Cl}_{2}$ for 3 times and then dried in a vacuum oven at $60^{\circ} \mathrm{C}$ for $24 \mathrm{~h}$.

\subsubsection{Preparation of PPy Coating}

The as-prepared hydrolyzed APEG-TPEs were immersed in ethyl alcohol and APS solution $(0.5 \mathrm{M})$ successively for a period of $10 \mathrm{~min}$. Then using filter papers to remove the surface liquid, the porous materials were located in a 1L-sized airtight container, followed by preheating it to $50{ }^{\circ} \mathrm{C}$. A plate loaded with $200 \mu \mathrm{L}$ of pyrrole was then placed near the porous materials to undergo the chemical vapor deposition for $30 \mathrm{~min}$. Subsequently, the PPy-coated porous materials was cleaned using DI water and desiccated in a vacuum oven overnight.

\subsubsection{Measurement of Solar Evaporation}

The solar irradiation was generated using a commercial Xenon lamp. A $4.8 \mathrm{~cm}-$ diameter glass beaker was filled with DI water and the $4 \mathrm{~cm}$-diameter APEG-TPEs was then located on the water-air interface. The simulated sunlight shed from the lamp irradiated on the porous material perpendicularly. In order to obtain a power density of solar irradiation of $1 \mathrm{~kW} \cdot \mathrm{m}^{-2}$, which was measured by solar power meter, the positions of the porous material and the Xenon lamp was controlled strictly. Using an electronic balance, the variation of mass of the system was measured every $5 \mathrm{~min}$. During measurement, the ambient temperature and relative humidity were controlled at $25 \pm 2{ }^{\circ} \mathrm{C}$ and $50 \pm 10 \%$, respectively. To obtain the equation of evaporation rate, $\mathrm{m}=\frac{\Delta \mathrm{m}}{\mathrm{S} \times \Delta \mathrm{t}}$, it could be calculated from the above measured experimental data of mass variation $\Delta \mathrm{m}$ of the system, the irritated surface area $\mathrm{S}$, and irradiation time $\Delta \mathrm{t}$ of the porous materials.

\subsubsection{Characterization}

SU-8010 (Hitachi, Tokyo, Japan) was used to obtain scanning electron microscopy (SEM) photograph. The solar absorbance of porous materials, A, was calculated from the equation, $\mathrm{A}=(1-\mathrm{R}-\mathrm{T}) \times 100 \%$, where $\mathrm{T}$ is the transmittance and $\mathrm{R}$ is the diffuse reflectance. In scope of a wavelength of 200-800 nm, the transmittance $\mathrm{T}$ and the diffuse reflectance $\mathrm{R}$ were measured by a UV-2450 spectrometer, which was equipped with an integration sphere (Shimadzu, Kyoto, Japan). Contact angle was captured using a microscope (JT-200M, Jingtuo Youcheng Technology Co., Ltd., Shenzhen, China) and measured by ImageJ software. Equipped with a thermal infrared camera accessory (FLIR ONE PRO, Flir System. Inc., Goleta, CA, USA), an iPhone was used to analyze and record the surface temperatures of the samples. Compressive tests were performed using Instron 5944 . The $15 \mathrm{~mm}$-diameter and $8 \mathrm{~mm}$-thick samples were used, and the loading rate was $0.1 \% \mathrm{~s}^{-1}$. Using a Netzsch laser flash apparatus (LFA 467), the thermal diffusivity was measured, and the thermal conductivity $\mathrm{k}\left(\mathrm{W} \cdot \mathrm{m}^{-1} \cdot \mathrm{K}^{-1}\right)$ was calculated according to the equation, 
$\mathrm{k}=\alpha \rho \mathrm{C}_{p}$, where $\alpha\left(\mathrm{mm}^{2} \cdot \mathrm{s}^{-1}\right)$ is the measured thermal diffusivity, $\rho\left(\mathrm{g} \cdot \mathrm{cm}^{-3}\right)$ is the density and $\mathrm{C}_{p}\left(\mathrm{~J} \cdot \mathrm{g}^{-1} \cdot \mathrm{K}^{-1}\right)$ is the heat capacity, and they were acquired in parallel through thermal diffusivity measurements by comparing with a standard reference.

\subsubsection{Simulations of Water Evaporation of APEG-TPEs with Different Porosity}

Simulations of water evaporation of APEG-TPEs with different porosity were carried out using COMSOL Multiphysics software. The model used was based on the model developed by Datta $[39,40]$. The model was established by referring to the relevant principles of the strong evaporation model [39] and the definition of model attributes [40]. In COMSOL software, dilute material transfer module was used to simulate the transfer process of liquid-state water and vapor-state water in porous materials, and laminar flow module was used to simulate the flow of wet air in the free-flowing area above. The heat transfer process was simulated by solid heat transfer module, and porous medium and wet air were added. Different from the rapid evaporation case of porous materials given by COMSOL software, a boundary heat source was set on the surface of the porous materials, and the effective power of the heat source was $1000 \mathrm{~W} \cdot \mathrm{m}^{-2}$. The moist air above the porous materials was allowed to flow freely over the boundary, regardless of horizontal gas flow. The area beneath the porous materials was large enough for liquid water to replenish in time. Therefore, the porous materials also had a mass transfer process for the liquid water beneath it, which is simulated by the dilute material transfer module. The values of each major parameter are shown in Table S1. The relationships between parameters were referred to the references $[40,41]$, in which the parameters of porous media were determined according to the experiments.

\section{Results and Discussion}

To balance thermal management and water transport, the integral solar evaporator is developed based on the interfacial engineering of thermal insulation porous materials. Thermal insulation porous materials are first prepared using attractive Pickering emulsion gels (APEGs) as templates. To prepare the APEGs, carboxyl-functionalized shellac nanoparticles were obtained by flash nanoprecipitation and they were dispersed in the water phase [42-44], and $\alpha, \omega$-diamino-functionalized polystyrene molecules $\left(\mathrm{NH}_{2}-\mathrm{PS}^{-\mathrm{NH}_{2}}\right)$ were dissolved in the photopolymerizable oil. By emulsifying the water phase in the oil phase, both shellac nanoparticles and telechelic polymers tend to adsorb at the oil/water interface to decrease the interfacial tension [45]. Each telechelic molecule with two amino end groups could simultaneously bind to two carboxyl-functionalized nanoparticles in two neighboring droplets via electrostatic attraction, forming a bridged droplet network. The APEG system consisted of bridged water droplets is thus established via the attractive electrostatic interactions between carboxyl-functionalized nanoparticles and $\alpha, \omega$-diaminofunctionalized telechelic polymers at the oil/water interface, as demonstrated in Figure 1a. Since the initiator is added in the oil phase, polymerization initiates in the oil phase when the APEGs are exposed to UV irradiation, yield APEG-templated porous evaporators (APEG-TPEs) with inter-connected pores. Since polystyrene is naturally a good thermal insulation material [38], APEG-TPEs are excellent scaffolds for the design of solar evaporators. The as-prepared APEG-TPEs are then hydrolyzed to improve water transport and a polypyrrole (PPy) layer is introduced on the top surface of the samples by chemical vapor deposition to realize photothermal conversion function, as schematically illustrated in Figure 1b. After the interfacial engineering, the APEG-TPEs integrate the three elements, i.e., thermal management, water transport and photothermal conversion, which make them an ideal solar evaporator, as modeled in Figure 1c. 

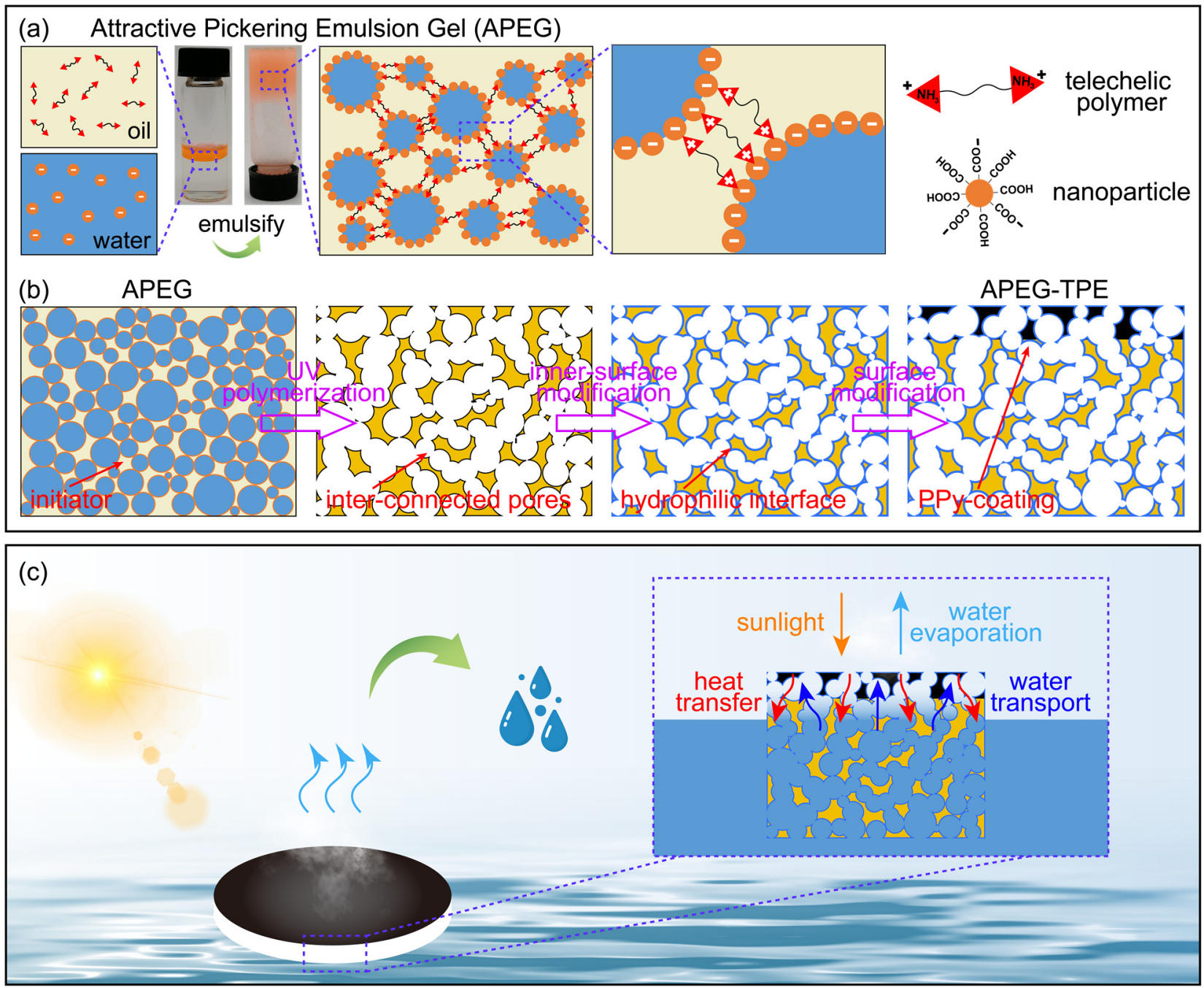

Figure 1. Attractive Pickering emulsion gel-templated porous evaporators (APEG-TPEs) for water distillation using solar energy. (a) Preparation of APEGs by bridging neighboring droplets using telechelic polymers via electrostatic interaction. (b) Preparation of APEG-TPEs through UV polymerization, inner-surface modification and surface modification. Hydrophilization and polypyrrole-coating are successively conducted to construct integrated APEG-TPEs for solar vapor generation. (c) Schematic illustration of water distillation using solar energy via APEG-TPEs.

One of the major advantages of APEGs is the versatility of changing the compositions. Styrene (St) and divinyl benzene (DVB) are chosen to prepare the scaffolds of the porous materials, which are the main components of commercialized thermal insulation polystyrene foams. However, because polystyrene is hydrophobic and thus adverse to water transport, $t$-butyl acrylate ( $t$-BA) is introduced as a co-monomer to improve the wettability of the porous materials, which could be post-hydrolyzed by trifluoroacetic acid to yield highly hydrophilic carboxylic acid groups on the surface, as schemed in Figure S1. The molar ratio among St, $t$-BA and DVB could be tuned in a wide range to tailor the mechanical properties of the APEG-templated porous materials, as summarized Table S2. The stress-strain measurements demonstrate that the compressive modulus increases as the fraction of DVB increases and decreases as the fraction of $t$-BA increases, as shown in Figure 2. To ensure good mechanical properties of the systems after polymerization and adequate $t$-BA for subsequent surface hydrolysis, $50 \% t$-BA, $40 \%$ St and $10 \%$ DVB are used as the oil phase to prepare APEG-TPEs. The as-prepared APEG-TPE is white in color with a low light absorption, as shown in Figure 3a. Cross-section of the sample reveals inter-connected pores, which build unobstructed pathways for water transport, as shown in the SEM images of Figure 3b. The surface properties of the samples before 
and after hydrolysis of $t$-BA are studied on model plain samples, as shown in Figure $3 \mathrm{c}, \mathrm{d}$, respectively. The static contact angle of water droplet on a pristine plain sample is $\sim 97^{\circ}$ and decreases to $\sim 64^{\circ}$ after hydrolysis, indicating successful transformation of $t$-butyl groups to carboxylic acid groups. Similar trends are observed in the dynamic contact angle of water droplet on APEG-TPE samples before and after hydrolysis, as shown in Figure 3e. Water droplet does not wet the $\mathrm{St} / \mathrm{t}$-BA/DVB porous materials and is nearly spherical on pristine APEG-TPEs with a contact angle of $\sim 150^{\circ}$, which is attributed to the hydrophobicity of the materials and the roughness of the porous structures. After hydrolysis, the contact angle of water droplet on hydrolyzed APEG-TPEs is substantially reduced to $\sim 100^{\circ}$.
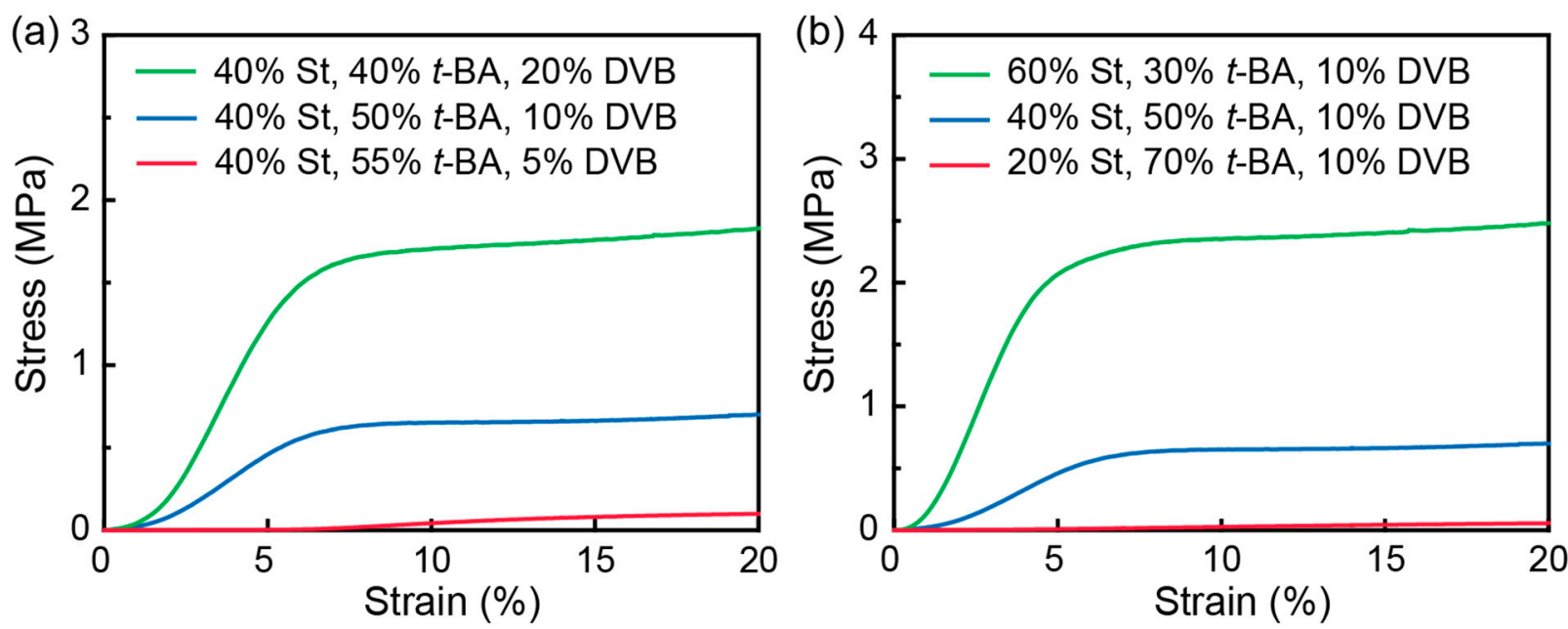

Figure 2. Tuning the mechanical properties of APEG-TPEs by adjusting the concentrations of styrene (St) monomers, $t$-butyl acrylate ( $t$-BA) monomers and divinyl benzene (DVB) cross-linkers. The compressive modulus (a) increases when the fraction of DVB increases or $(\mathbf{b})$ when the fraction of $t$-BA decreases.

The ability of solar absorption of the APEG-TPEs plays a predominant role in the performance of solar evaporation. A photothermal conversion layer of polypyrrole (PPy) is thus introduced on the top surface of hydrolyzed APEG-TPEs by chemical vapor deposition. Pristine APEG-TPEs present a relatively low absorbance of $~ 15 \%$ in the visible light range, as shown in Figure 3f. PPy-coated APEG-TPEs demonstrate a high absorbance up to $\sim 97 \%$ in the whole measured spectrum, which is comparable to the high performance of a porous plasmonic absorber as reported in [16]. To assess the performances of photothermal conversion, the surface temperatures of the samples under simulated sunlight are measured by a thermal infrared camera. When the samples are placed in air, the surface temperature of PPy-coated APEG-TPEs is $\sim 57^{\circ} \mathrm{C}$ upon sun irradiation, and it is much higher than that of pristine APEG-TPEs of $\sim 29^{\circ} \mathrm{C}$, as shown in Figure $3 \mathrm{~g}$. When the samples are floating on water, PPy-coated APEG-TPEs also exhibit a higher surface temperature of $\sim 38^{\circ} \mathrm{C}$ than that of pristine APEG-TPEs of $\sim 28^{\circ} \mathrm{C}$. These results suggest that PPy-coated APEG-TPEs have excellent solar absorption performances and are promising candidates for solar evaporators.

PPy-coated APEG-TPEs are black, while pristine APEG-TPEs are white, as shown in Figure $4 a, b$, respectively. The performances of solar evaporation of the APEG-TPE samples are tested by placing them on a water surface under simulated solar irradiation. The weight loss of water over time is recorded using a balance. All APEG-TPE samples with a diameter of $4 \mathrm{~cm}$ are able to float on the water surface. The weight losses of water using different samples and their corresponding evaporation rates over irradiation time are recorded in Figure 4c,d, respectively. As a control, the evaporation rates of water with APEG-TPEs in darkness is as low as $0.10 \mathrm{~kg} \cdot \mathrm{m}^{-2} \cdot \mathrm{h}^{-1}$, as shown in Figure S2. When pristine APEG-TPEs are employed as the solar evaporators under simulated solar irradiation, the water evaporation rate reaches a value of $\sim 0.37 \mathrm{~kg} \cdot \mathrm{m}^{-2} \cdot \mathrm{h}^{-1}$, which is very close to the value of water evaporation from neat air/water interface under the 
same condition. In contrast, PPy-coated APEG-TPEs could significantly improve the solar evaporation performances with an equilibrium water evaporation rate of $\sim 1.0 \mathrm{~kg} \cdot \mathrm{m}^{-2} \cdot \mathrm{h}^{-1}$. When water pathways are hydrolyzed to facilitate water transport for water evaporation, PPy-coated hydrolyzed APEG-TPEs could further increase the water evaporation rate to $\sim 1.1 \mathrm{~kg} \cdot \mathrm{m}^{-2} \cdot \mathrm{h}^{-1}$, which is higher than value of $\sim 0.8 \mathrm{~kg} \cdot \mathrm{m}^{-2} \cdot \mathrm{h}^{-1}$ in PPy-coated vertically oriented porous membranes [24].
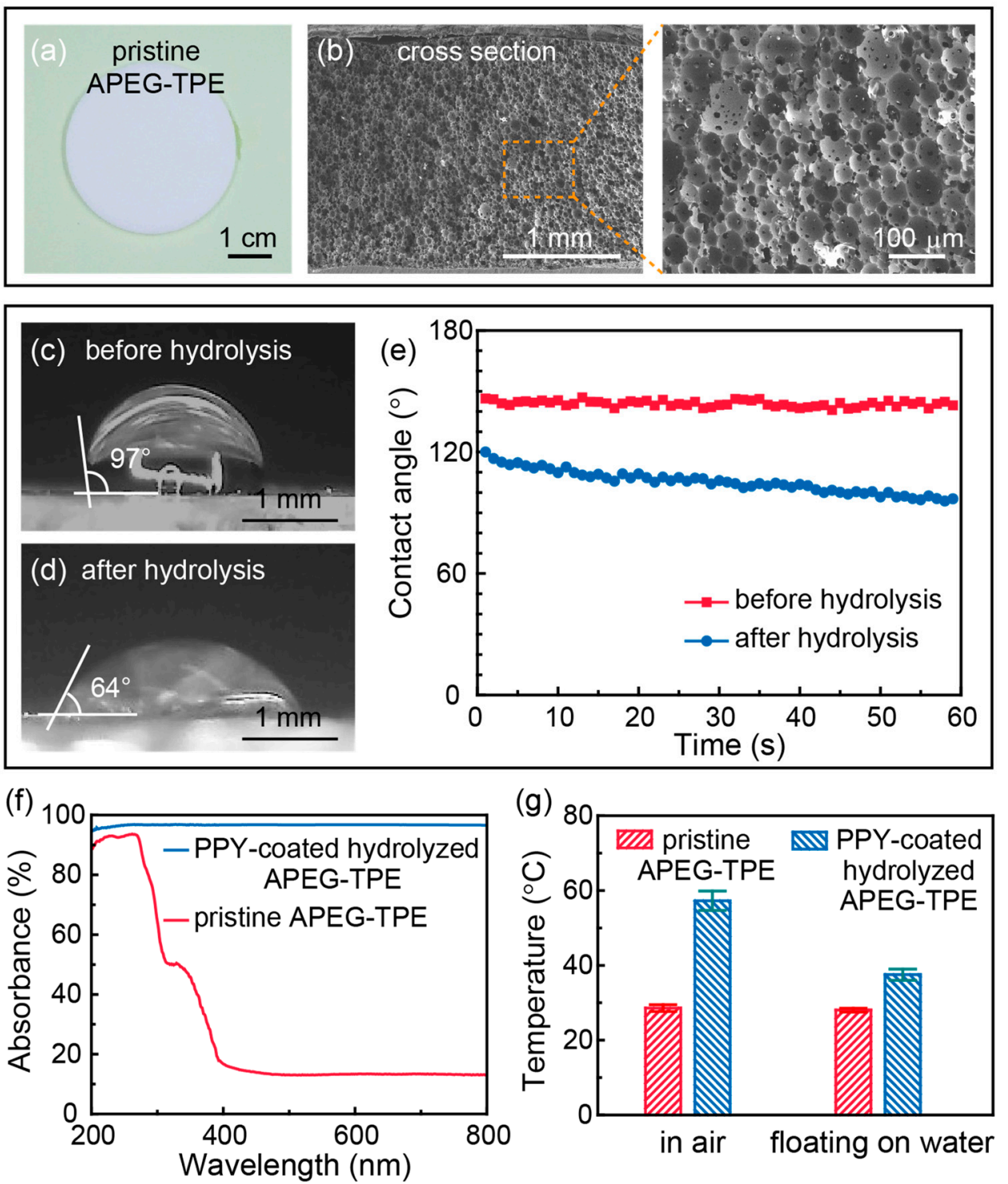

Figure 3. Optimizations of APEG-TPEs. (a) Opitical and (b) SEM images of pristine APEG-TPEs, showing inter-connected pores in the cross section. If not specified, the oil-to-water ratio of APEGs is $1: 9$ and the oil phase consists of $40 \%$ styrene, $50 \% t$-butyl acrylate and $10 \%$ divinyl benzene. (c) and (d) Static and (e) dynamic contact angles of water droplets on plain samples before and after hydrolysis of $t$-BA. (f) Light absorption of pristine APEG-TPEs and PPy-coated hydrolyzed APEGTPEs. (g) Equilibrium surface temperatures of pristine APEG-TPEs and PPy-coated hydrolyzed APEG-TPEs in air or floating on water after 1 hour's irradiation of simulated sunlight. 

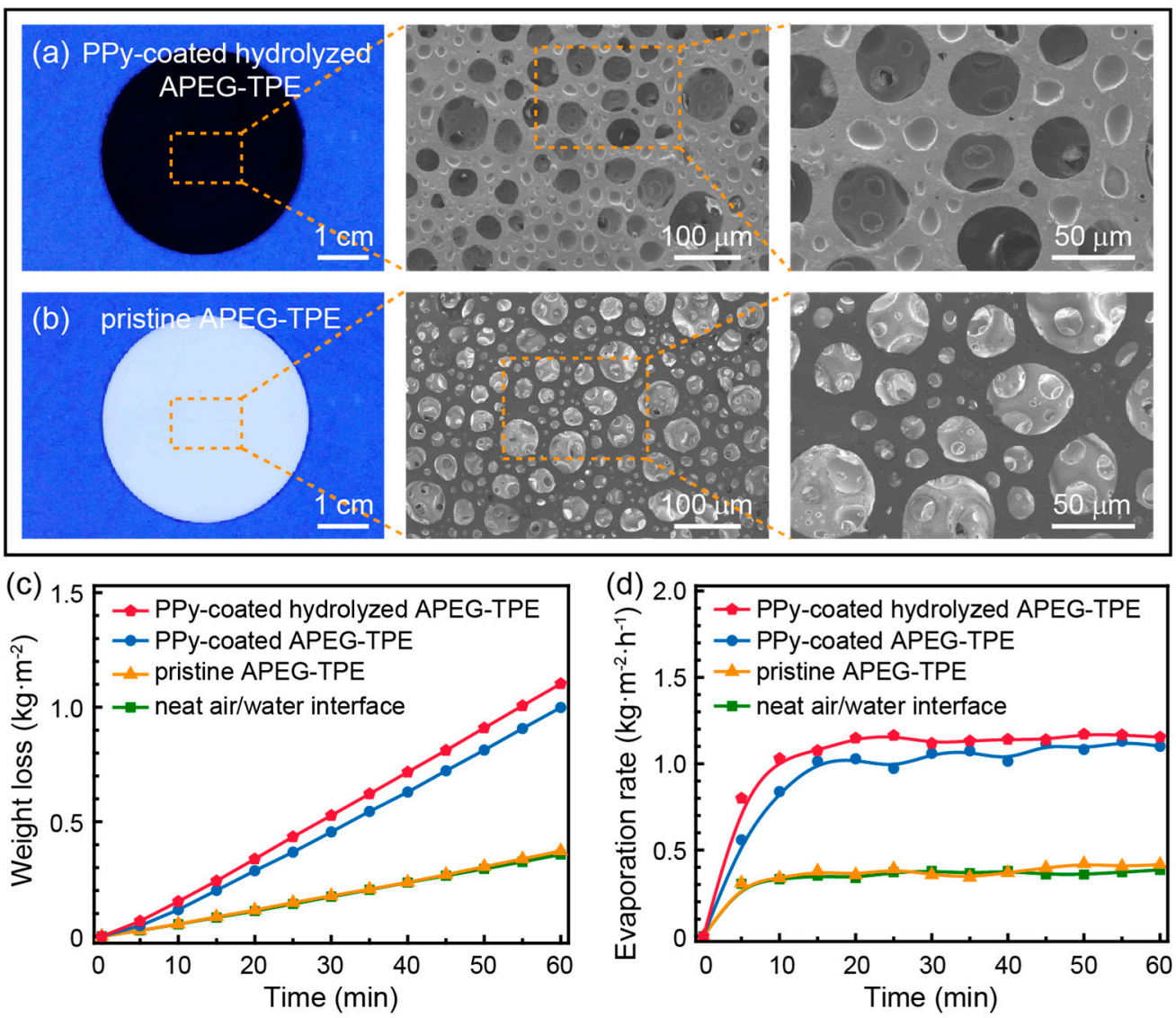

Figure 4. Performances of APEG-TPEs for solar vapor generation. Optical and SEM images of (a) PPy-coated hydrolyzed APEG-TPEs and (b) pristine APEG-TPEs. (c) Accumulative water losses and (d) evaporation rates of different APEG-TPEs as a function of irradiation time.

To quantitively describe the efficiency of absorbed solar energy for water evaporation, the solar evaporation efficiency $\eta$ is calculated by the formula [24], $\eta=\frac{\left(m-m_{0}\right) H_{v}}{C_{o p t} P_{0}}$, where $m$ is the water evaporation rate under simulated solar irradiation, $m_{0}$ is the water evaporation rate in darkness, $H_{v}$ is the enthalpy of liquid-to-vapor phase change $\left(\sim 2260 \mathrm{~kJ} \cdot \mathrm{kg}^{-1}\right), C_{o p t}=1$ is the optical concentration, and $P_{0}$ is the power density of simulated solar irradiation $\left(1 \mathrm{~kW} \cdot \mathrm{m}^{-2}\right)$. The solar evaporation efficiency of pristine APEG-TPEs under simulated solar irradiation is only $\sim 23 \%$, while PPy-coated hydrolyzed APEG-TPEs could reach up to $\sim 69 \%$, as summarized in Table S3. The optimized solar evaporation efficiency of PPycoated hydrolyzed APEG-TPEs is two times higher than that of pristine APEG-TPEs under simulated solar irradiation, which is also as high as the that of a photothermal membrane distillation that combines solar harvesting and heat localization [3].

To systematically investigate the performances of PPy-coated hydrolyzed APEG-TPEs on the porosity of the materials, APEG-TPEs with different porosity of $80 \%, 85 \%$ and $90 \%$ are prepared by changing the oil-to-water ratio, as shown in Figure 5a and Figure S3. The density of pores increases slightly as the porosity increases and the APEG-TPEs of different porosity are hydrolyzed and coated following the same procedure as above. After hydrolysis and PPy-coating, the performances of solar evaporation by the samples are compared under simulated solar irradiation, as shown in Figure $5 b, c$. The water evaporation rates of PPy-coated hydrolyzed APEG-TPEs with porosity of $80 \%, 85 \%$ and $90 \%$ are $\sim 0.76, \sim 0.94$ and $\sim 1.10 \mathrm{~kg} \cdot \mathrm{m}^{-2} \cdot \mathrm{h}^{-1}$, respectively; their corresponding solar evaporation efficiencies are $48 \%, 59 \%$ and $69 \%$, as summarized in Table S4. The results suggest that APEG-TPEs with a higher porosity present a better solar evaporation performance. The enhancement in solar evaporation performances of APEG-TPEs with a higher porosity is attributed to enhanced water transport. The dependence of solar evaporation performances 
on porosity is further confirmed by simulation using COMSOL Multiphysics software; the simulation results demonstrate good agreement with the experiments, as shown in Figure 5d,e.

(a) porosity $=80 \%$

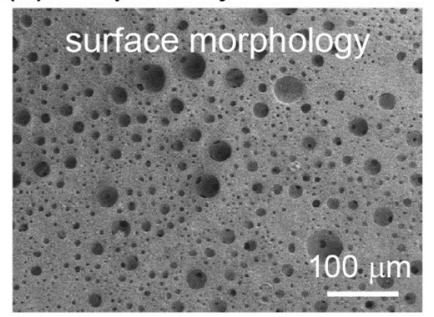

(b)

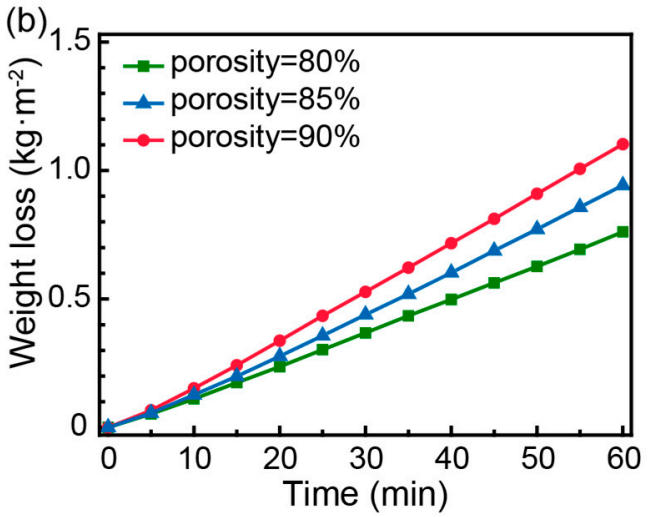

(d)

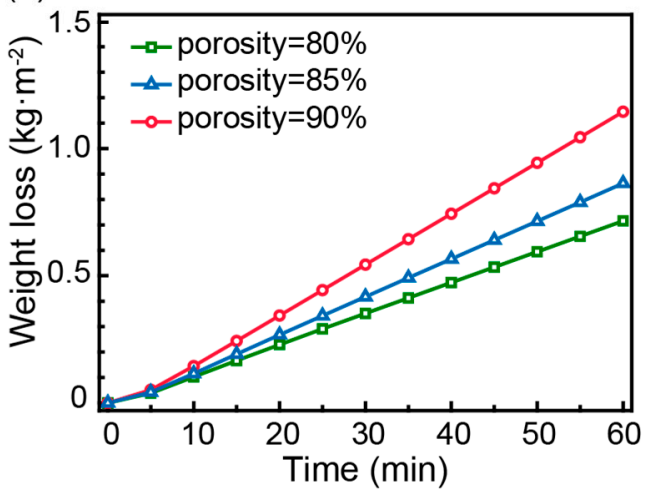

porosity $=85 \%$

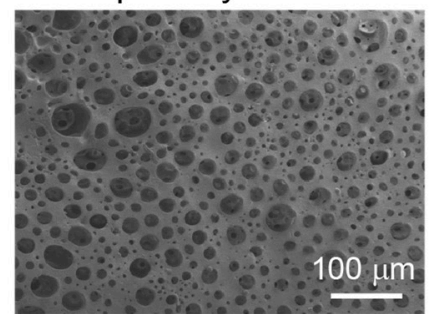

porosity $=90 \%$

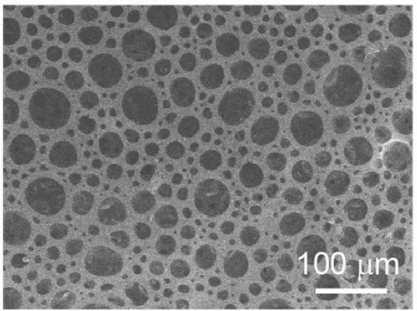

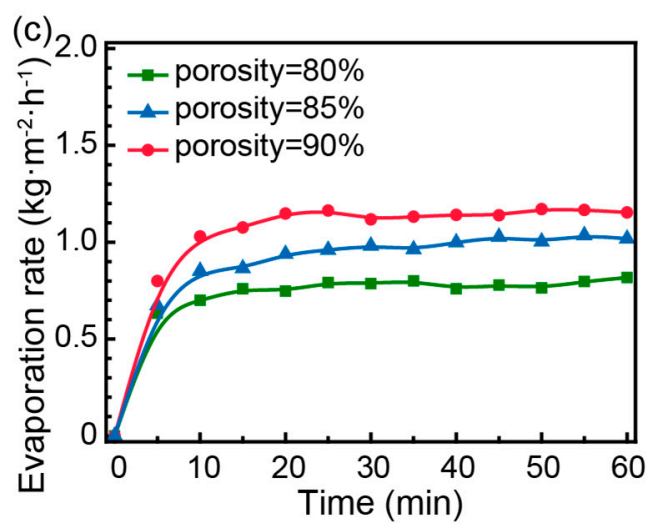

(e)

COMSOL simulation

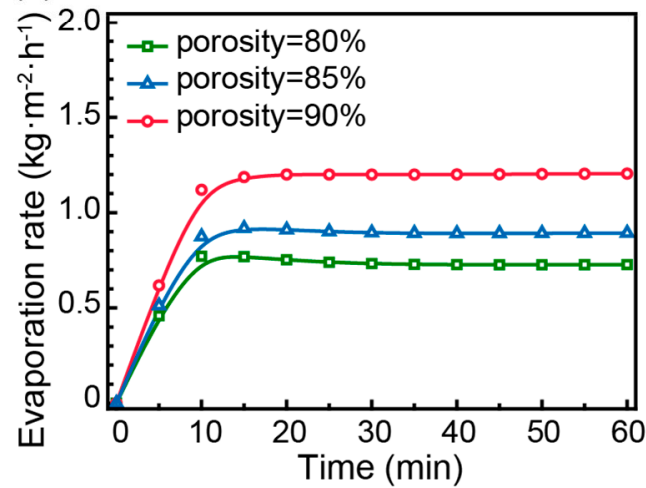

Figure 5. Effects of porosity on the performances of water evaporation using APEG-TPEs. (a) SEM images of APEG-TPEs with a porosity $=80 \%, 85 \%$ and $90 \%$, respectively. (b) Accumulative water losses and (c) evaporation rates of APEG-TPEs with different porosity as a function of irradiation time. COMSOL simulations of (d) accumulative water losses and (e) evaporation rates of APEG-TPEs with different porosity as a function of irradiation time.

\section{Conclusions}

In summary, solar evaporators are designed using attractive Pickering emulsion gels (APEGs) as templates. APEG-templated porous evaporators (APEG-TPEs) are excellent thermal insulation materials. The compositions of APEG-TPEs could be designed to enable post hydrolysis for enhanced water transport and PPy-coating is introduced by chemical vapor deposition to improve photothermal conversion performances. After interfacial engineering, PPy-coated hydrolyzed APEG-TPEs exhibit a high light absorption of 97\%. The porosity of APEG-TPEs is tailored to realize optimal performances of photothermal conversion, water transport and thermal management, reaching a water evaporation rate of $1.1 \mathrm{~kg} \cdot \mathrm{m}^{-2} \cdot \mathrm{h}^{-1}$ under simulated solar irradiation and a solar evaporation efficiency up to $\sim 69 \%$. These results provide rational designs of APEG-TPEs with high performances and hold great promise for scale up due to the versatile fabrication of APEG-TPEs. 
Supplementary Materials: The following are available online at https:/ / www.mdpi.com/article/10 $.3390 /$ en14196077/s1. Table S1: The values of major parameters in Simulations of water evaporation of APEG-TPEs. Table S2: Tunable mechanical properties of porous materials prepared using APEGs as templates. Table S3: Performances of water evaporation using interface-engineered APEG-TPE samples. Table S4: Performances of water evaporation using APEG-TPE samples with different porosity. Figure S1: Schematic illustration of the hydrolysis of $t$-BA. Figure S2: Water evaporation in dark from neat air/water interface or using APEG-TPEs as control groups. Figure S3: Optical images of APEG-TPEs with different porosities.

Author Contributions: Conceptualization, X.Y. and D.C.; methodology, B.W.; software, Q.W.; validation, X.Y., B.W. and L.C.; formal analysis, X.Y.; investigation, B.W.; resources, D.C.; data curation, B.W.; writing — original draft preparation, X.Y.; writing—review and editing, L.C.; visualization, Q.W.; supervision, F.Y.; project administration, X.Y.; funding acquisition, D.C. All authors have read and agreed to the published version of the manuscript.

Funding: This research was funded by Zhejiang Provincial Natural Science Foundation of China with grant number Y20B060027, National Natural Science Foundation of China with grant number 21878258, National Key Research and Development Program of China with grant number 2020YFA0908200, and Innovation Project of Keqiao.

Institutional Review Board Statement: Not applicable.

Informed Consent Statement: Not applicable.

Data Availability Statement: Not applicable.

Conflicts of Interest: The authors declare no conflict of interest.

\section{References}

1. Lu, Y.; Song, S.; Wang, R.; Liu, Z.; Meng, J.; Sweetman, A.J.; Jenkins, A.; Ferrier, R.C.; Li, H.; Luo, W.; et al. Impacts of Soil and Water Pollution on Food Safety and Health Risks in China. Environ. Int. 2015, 77, 5-15. [CrossRef]

2. Zhao, J.; Gleason, K.K. Solvent-Less Vapor-Phase Fabrication of Membranes for Sustainable Separation Processes. Engineering 2020, 6, 1432-1442. [CrossRef]

3. Gong, B.; Yang, H.; Wu, S.; Yan, J.; Cen, K.; Bo, Z.; Ostrikov, K.K. Superstructure-Enabled Anti-Fouling Membrane for Efficient Photothermal Distillation. ACS Sustain. Chem. Eng. 2019, 7, 20151-20158. [CrossRef]

4. Wu, L.; Dong, Z.; Cai, Z.; Ganapathy, T.; Fang, N.X.; Li, C.; Yu, C.; Zhang, Y.; Song, Y. Highly Efficient Three-Dimensional Solar Evaporator for High Salinity Desalination by Localized Crystallization. Nat. Commun. 2020, 11, 521. [CrossRef] [PubMed]

5. Bai, H.; Zhao, T.; Cao, M. Interfacial Solar Evaporation for Water Production: From Structure Design to Reliable Performance. Mol. Syst. Des. Eng. 2020, 5, 419-432. [CrossRef]

6. Zhu, L.; Gao, M.; Peh, C.K.N.; Ho, G.W. Recent Progress in Solar-Driven Interfacial Water Evaporation: Advanced Designs and Applications. Nano Energy 2019, 57, 507-518. [CrossRef]

7. Tao, P.; Ni, G.; Song, C.; Shang, W.; Wu, J.; Zhu, J.; Chen, G.; Deng, T. Solar-Driven Interfacial Evaporation. Nat. Energy 2018, 3, 1031-1041. [CrossRef]

8. Lin, Y.; Xu, H.; Shan, X.; Di, Y.; Zhao, A.; Hu, Y.; Gan, Z. Solar Steam Generation Based on the Photothermal Effect: From Designs to Applications, and Beyond. J. Mater. Chem. A 2019, 7, 19203-19227. [CrossRef]

9. Cao, S.; Jiang, Q.; Wu, X.; Ghim, D.; Derami, H.G.; Chou, P.-I.; Jun, Y.-S.; Singamaneni, S. Advances in Solar Evaporator Materials for Freshwater Generation. J. Mater. Chem. A 2019, 7, 24092-24123. [CrossRef]

10. Mirmanto; Sayoga, I.M.A.; Wijayanta, A.T.; Sasmito, A.P.; Aziz, M. Enhancement of Continuous-Feed Low-Cost Solar Distiller: Effects of Various Fin Designs. Energies 2021, 14, 4844. [CrossRef]

11. Zhou, L.; Li, X.; Ni, G.W.; Zhu, S.; Zhu, J. The Revival of Thermal Utilization from the Sun: Interfacial Solar Vapor Generation. Natl. Sci. Rev. 2019, 6, 562-578. [CrossRef]

12. Zhao, F.; Guo, Y.; Zhou, X.; Shi, W.; Yu, G. Materials for Solar-Powered Water Evaporation. Nat. Rev. Mater. $2020,5,388-401$. [CrossRef]

13. Chen, L.; Yang, C.; Xiao, Y.; Yan, X.; Hu, L.; Eggersdorfer, M.; Chen, D.; Weitz, D.; Ye, F. Millifluidics, Microfluidics, and Nanofluidics: Manipulating Fluids at Varying Length Scales. Mater. Today Nano 2021, 16, 100136. [CrossRef]

14. Alvi, J.Z.; Feng, Y.; Wang, Q.; Imran, M.; Khan, L.A.; Pei, G. Effect of Phase Change Material Storage on the Dynamic Performance of a Direct Vapor Generation Solar Organic Rankine Cycle System. Energies 2020, 13, 5904. [CrossRef]

15. Zhou, L.; Tan, Y.; Ji, D.; Zhu, B.; Zhang, P.; Xu, J.; Gan, Q.; Yu, Z.; Zhu, J. Self-Assembly of Highly Efficient, Broadband Plasmonic Absorbers for Solar Steam Generation. Sci. Adv. 2016, 2, e1501227. [CrossRef] [PubMed]

16. Zhou, L.; Tan, Y.; Wang, J.; Xu, W.; Yuan, Y.; Cai, W.; Zhu, S.; Zhu, J. 3D Self-Assembly of Aluminium Nanoparticles for Plasmon-Enhanced Solar Desalination. Nat. Photonics 2016, 10, 393-398. [CrossRef] 
17. Yang, Y.; Zhao, H.; Yin, Z.; Zhao, J.; Yin, X.; Li, N.; Yin, D.; Li, Y.; Lei, B.; Du, Y. A General Salt-Resistant Hydrophilic/Hydrophobic Nanoporous Double Layer Design for Efficient and Stable Solar Water Evaporation Distillation. Mater. Horiz. 2018, 5, 1143-1150. [CrossRef]

18. Wu, T.; Li, H.; Xie, M.; Shen, S.; Wang, W.; Zhao, M.; Mo, X.; Xia, Y. Incorporation of Gold Nanocages into Electrospun Nanofibers for Efficient Water Evaporation through Photothermal Heating. Mater. Today Energy 2019, 12, 129-135. [CrossRef]

19. Chen, Z.; Jin, L.; Hao, W.; Ren, W.; Cheng, H.-M. Synthesis and Applications of Three-Dimensional Graphene Network Structures. Mater. Today Nano 2019, 5, 100027. [CrossRef]

20. Zhao, Y.; Yuan, H.; Zhang, X.; Xue, G.; Tang, J.; Chen, Y.; Zhang, X.; Zhou, W.; Liu, H. Laser-Assisted Synthesis of Cobalt@NDoped Carbon Nanotubes Decorated Channels and Pillars of Wafer-Sized Silicon as Highly Efficient Three-Dimensional Solar Evaporator. Chin. Chem. Lett. 2021. [CrossRef]

21. Chen, W.; Chen, S.; Liang, T.; Zhang, Q.; Fan, Z.; Yin, H.; Huang, K.-W.; Zhang, X.; Lai, Z.; Sheng, P. High-Flux Water Desalination with Interfacial Salt Sieving Effect in Nanoporous Carbon Composite Membranes. Nat. Nanotechnol. 2018, 13, 345-350. [CrossRef] [PubMed]

22. Ogieglo, W.; Puspasari, T.; Hota, M.K.; Wehbe, N.; Alshareef, H.N.; Pinnau, I. Nanohybrid Thin-Film Composite Carbon Molecular Sieve Membranes. Mater. Today Nano 2020, 9, 100065. [CrossRef]

23. Zhang, C.; Wu, M.-B.; Wu, B.-H.; Yang, J.; Xu, Z.-K. Solar-Driven Self-Heating Sponges for Highly Efficient Crude Oil Spill Remediation. J. Mater. Chem. A 2018, 6, 8880-8885. [CrossRef]

24. Yu, H.-H.; Yan, L.-J.; Shen, Y.-C.; Chen, S.-Y.; Li, H.-N.; Yang, J.; Xu, Z.-K. Janus Poly(Vinylidene Fluoride) Membranes with Penetrative Pores for Photothermal Desalination. Research 2020, 2020, 1-11. [CrossRef] [PubMed]

25. Li, S.; He, Y.; Guan, Y.; Liu, X.; Liu, H.; Xie, M.; Zhou, L.; Wei, C.; Yu, C.; Chen, Y. Cellulose Nanofibril-Stabilized Pickering Emulsion and in Situ Polymerization Lead to Hybrid Aerogel for High-Efficiency Solar Steam Generation. ACS Appl. Polym. Mater. 2020, 2, 4581-4591. [CrossRef]

26. Huang, S.; Li, Q.; Xiao, Y.; Chen, X.; Cui, Y.; Meng, X.; Hu, X.; Liu, Z.; Li, C.; Zhang, C. Improvement in Power Conversion Efficiency of All-Polymer Solar Cells Enabled by Ultrafast Channels for Charge Dynamics. Mater. Today Nano 2021, 100133. [CrossRef]

27. Wu, S.; Xiong, G.; Yang, H.; Tian, Y.; Gong, B.; Wan, H.; Wang, Y.; Fisher, T.S.; Yan, J.; Cen, K. Scalable Production of Integrated Graphene Nanoarchitectures for Ultrafast Solar-Thermal Conversion and Vapor Generation. Matter 2019, 1, 1017-1032. [CrossRef]

28. Wu, S.; Xiong, G.; Yang, H.; Gong, B.; Tian, Y.; Xu, C.; Wang, Y.; Fisher, T.; Yan, J.; Cen, K. Multifunctional Solar Waterways: PlasmaEnabled Self-Cleaning Nanoarchitectures for Energy-Efficient Desalination. Adv. Energy Mater. 2019, 9, 1901286. [CrossRef]

29. Wu, S.-L.; Chen, H.; Wang, H.-L.; Chen, X.; Yang, H.-C.; Darling, S.B. Solar-Driven Evaporators for Water Treatment: Challenges and Opportunities. Environ. Sci. Water Res. Technol. 2021, 7, 24-39. [CrossRef]

30. Xu, W.; Xing, Y.; Liu, J.; Wu, H.; Cui, Y.; Li, D.; Guo, D.; Li, C.; Liu, A.; Bai, H. Efficient Water Transport and Solar Steam Generation via Radially, Hierarchically Structured Aerogels. ACS Nano 2019, 13, 7930-7938. [CrossRef] [PubMed]

31. Li, X.; Xu, W.; Tang, M.; Zhou, L.; Zhu, B.; Zhu, S.; Zhu, J. Graphene Oxide-Based Efficient and Scalable Solar Desalination under One Sun with a Confined 2D Water Path. Proc. Natl. Acad. Sci. USA 2016, 113, 13953-13958. [CrossRef] [PubMed]

32. Gao, T.; Li, Y.; Chen, C.; Yang, Z.; Kuang, Y.; Jia, C.; Song, J.; Hitz, E.M.; Liu, B.; Huang, H. Architecting a Floatable, Durable, and Scalable Steam Generator: Hydrophobic/Hydrophilic Bifunctional Structure for Solar Evaporation Enhancement. Small Methods 2019, 3, 1800176. [CrossRef]

33. Xu, N.; Hu, X.; Xu, W.; Li, X.; Zhou, L.; Zhu, S.; Zhu, J. Mushrooms as Efficient Solar Steam-Generation Devices. Adv. Mater. 2017, 29, 1606762. [CrossRef] [PubMed]

34. Guo, Y.; Zhao, X.; Zhao, F.; Jiao, Z.; Zhou, X.; Yu, G. Tailoring Surface Wetting States for Ultrafast Solar-Driven Water Evaporation. Energy Environ. Sci. 2020, 13, 2087-2095. [CrossRef]

35. Wu, B.; Sun, Z.; Wu, J.; Ruan, J.; Zhao, P.; Liu, K.; Zhao, C.; Sheng, J.; Liang, T.; Chen, D. Nanoparticle-Stabilized Oxygen Microcapsules Prepared by Interfacial Polymerization for Enhanced Oxygen Delivery. Angew. Chem. Int. Ed. 2021, 60, 9284-9289. [CrossRef]

36. Chen, L.; Xiao, Y.; Wu, Q.; Yan, X.; Zhao, P.; Ruan, J.; Shan, J.; Chen, D.; Weitz, D.A.; Ye, F. Emulsion Designer Using Microfluidic Three-Dimensional Droplet Printing in Droplet. Small 2021, 2102579. [CrossRef] [PubMed]

37. Sun, Z.; Wu, B.; Ren, Y.; Wang, Z.; Zhao, C.-X.; Hai, M.; Weitz, D.A.; Chen, D. Diverse Particle Carriers Prepared by CoPrecipitation and Phase Separation: Formation and Applications. ChemPlusChem 2021, 86, 49-58. [CrossRef]

38. Wu, B.; Yang, C.; Xin, Q.; Kong, L.; Eggersdorfer, M.; Ruan, J.; Zhao, P.; Shan, J.; Liu, K.; Chen, D.; et al. Attractive Pickering Emulsion Gels. Adv. Mater. 2021, 2102362. [CrossRef] [PubMed]

39. Datta, A.K. Porous Media Approaches to Studying Simultaneous Heat and Mass Transfer in Food Processes. I: Problem Formulations. J. Food Eng. 2007, 80, 80-95. [CrossRef]

40. Datta, A.K. Porous Media Approaches to Studying Simultaneous Heat and Mass Transfer in Food Processes. II: Property Data and Representative Results. J. Food Eng. 2007, 80, 96-110. [CrossRef]

41. Evaporation in Porous Media with Large Evaporation Rates. Available online: http://cn.comsol.com/model/evaporation-inporous-media-with-large-evaporation-rates-33731 (accessed on 21 April 2021). 
42. Kong, L.; Chen, R.; Wang, X.; Zhao, C.-X.; Chen, Q.; Hai, M.; Chen, D.; Yang, Z.; Weitz, D.A. Controlled Co-Precipitation of Biocompatible Colorant-Loaded Nanoparticles by Microfluidics for Natural Color Drinks. Lab Chip 2019, 19, $2089-2095$. [CrossRef] [PubMed]

43. Sun, Z.; Yang, C.; Wang, F.; Wu, B.; Shao, B.; Li, Z.; Chen, D.; Yang, Z.; Liu, K. Biocompatible and PH-Responsive Colloidal Surfactants with Tunable Shape for Controlled Interfacial Curvature. Angew. Chem. Int. Ed. 2020, 59, 9365-9369. [CrossRef] [PubMed]

44. Wu, B.; Yang, C.; Li, B.; Feng, L.; Hai, M.; Zhao, C.-X.; Chen, D.; Liu, K.; Weitz, D.A. Active Encapsulation in Biocompatible Nanocapsules. Small 2020, 16, 2002716. [CrossRef] [PubMed]

45. Sun, Z.; Yang, C.; Eggersdorfer, M.; Cui, J.; Li, Y.; Hai, M.; Chen, D.; Weitz, D.A. A General Strategy for One-Step Fabrication of Biocompatible Microcapsules with Controlled Active Release. Chin. Chem. Lett. 2020, 31, 249-252. [CrossRef] 